\title{
Effect of Individualized Dietary Intervention on Oxidative Stress in Patients with Type 2 Diabetes Complicated by Tuberculosis in Xinjiang, China
}

\author{
Liyun He - Guoming Zhang • Meng Wei • Yanjun Zhao • \\ Weixing Chen · Qiaojun Peng · Guiyun Meng
}

Received: March 7, 2019 / Published online: September 19, 2019

(C) The Author(s) 2019

\begin{abstract}
Introduction: This study aimed to examine the effect of individual dietary intervention on nutrition and oxidative stress of patients with type 2 diabetes mellitus (T2DM) complicated by pulmonary tuberculosis (PTB) in Xinjiang.

Methods: A total of 164 patients with T2DM and PTB from January 2018 to December 2018 in the Chest Hospital of Xinjiang Uyghur Autonomous Region were included. According to the random number table method, the patients were divided into an experimental group $(n=82)$ and control group $(n=82)$. The original treatments of PTB and T2DM were maintained. The control group was treated with a self-controlled diet, while the experimental group was treated with an individual
\end{abstract}

Liyun He and Guoming Zhang are the co-first authors.

Enhanced Digital Features To view enhanced digital features for this article go to https://doi.org/10.6084/ m9.figshare.9778511.

L. He · G. Zhang · M. Wei · Y. Zhao · Q. Peng ( $₫)$ The First Affiliated Hospital of Xinjiang Medical University, Urumqi 830000, Xinjiang,

People's Republic of China

e-mail: 1369157578@qq.com

W. Chen · G. Meng ( $₫)$

Chest Hospital of Xinjiang Uyghur Autonomous

Region, Urumqi 830000, Xinjiang,

People's Republic of China

e-mail: 2669560179@qq.com quantitative dietary intervention. All patients included in our study were closely followed up for 3 months and the serum levels of variables related to nutrition, oxidative stress, and blood glucose were measured to compare each patient's nutritional and oxidative stress after the intervention. Proportions of patients with sputum testing positive for bacteria before and after the intervention were also recorded and compared between the two groups.

Results: There were no significant differences between the two groups with regard to age, sex, labor intensity, marital status, course and treatment of diabetes, and proportion of patients with sputum positive for bacteria before the intervention $(P>0.05)$. There were statistically significant differences between the two groups in variables to related nutrition and oxidative stress, especially SOD which was increased by $13.2 \%$ in the experimental group and $2.5 \%$ in the control group after intervention $(P<0.05)$. The proportion of patients with sputum positive for bacteria in the experimental group was significantly lower than that in the control group, and the difference was statistically significant $(P<0.05)$.

Conclusion: A combination of individual quantitative dietary interventions can improve the nutritional status of patients with T2DM-PTB and adjust the oxidative stress. This method provides a theoretical basis for a reasonable diet in patients with T2DM-PTB. 
Trial Registration: ChiCTR900025477 (Retrospectively registered).

Keywords: Dietary intervention; Pulmonary tuberculosis; Type 2 diabetes

\section{INTRODUCTION}

Diabetes is a metabolic endocrine disease. Latest data from the World Diabetes Foundation (WDF) (2015) show that the number of adult patients with diabetes worldwide is expected to increase from 415 million to 642 million by 2040 , with the majority of patients in developing countries [1]. The prevalence of type 2 diabetes mellitus (T2DM) among Xinjiang residents was $14 \%$; this is much higher than the average prevalence in China [2]. Pulmonary tuberculosis (PTB) is a chronic pulmonary infectious disease caused by Mycobacterium tuberculosis (MTB) complex flora and is often accompanied by varying degrees of malnutrition. Latest data show that tuberculosis prevalence is still very high in China, ranking third in the world behind India and Indonesia [3]. At present, approximately 500 million people are infected with MTB in China, and approximately 1.5 million new cases are reported every year [4]. Tuberculosis shows the characteristics of high infection, morbidity, and drug resistance rates in China [5].

Patients with T2DM are at high risk of PTB infection [6]. PTB is a common complication of T2DM [7], and it can aggravate the glucose metabolism disorder in patients with T2DM, increase the incidence of ketoacidosis, and the prognosis is poor. Patients with T2DM have increased glucose levels in blood and tissues, impairing protein and fat metabolism, which is conducive to the growth and reproduction of MTB, and more susceptible to multiple drugresistant strains, which worsens the condition of patients with PTB [8]. In patients with T2DM-PTB, there are often systemic metabolic disorders, acid-base imbalance, negative nitrogen balance, and malnutrition; protein catabolism is increased significantly, resulting in excessive protein consumption, reduced nutritional intake, reduced protein synthesis and metabolism, and protein-energy malnutrition (PEM) is prone to occur. The incidence of PEM is 42.1-86.3\% [9]. The prognosis of PEM depends first on whether T2DM is controlled, and secondly on the severity of PTB. There are homogeneity and differences in dietary treatment. Dietary control of patients with T2DM should take into account the nutritional needs of PTB. On the contrary, the nutritional conditions of PTB should be adjusted under the conditions of caloric balance of T2DM.

This prospective controlled study aimed to examine the nutritional and oxidative stress status of patients with T2DM-PTB in Xinjiang. It will assist nutritionists in formulating personalized diets for patients according to patients' preferences. The purpose is to achieve the goal of enhancing patients' resistance and immunity, reducing adverse outcomes, and improving survival rate and quality of life.

\section{METHODS}

\section{Ethics}

A total of 164 patients with T2DM-PTB were enrolled in this study in the Thoracic Hospital of Xinjiang Uyghur Autonomous Region from January 2018 to December 2018. All selected patients voluntarily participated in the study. The study was examined and approved by the Ethics Committee of the First Affiliated Hospital of Xinjiang Medical University. All procedures performed in studies involving human participants were in accordance with the ethical standards of our local ethics committee (the Ethics Committee of the First Affiliated Hospital of Xinjiang Medical University) and with the 1964 Helsinki declaration and its later amendments or comparable ethical standards. Informed consent was obtained from all individual participants included in the study. Prior to providing informed consent, all subjects were informed of the study objectives and the risks and benefits of the study. All subjects were given sufficient time to decide whether they would like to participate in the trial. 


\section{Inclusion and Exclusion Criteria}

The inclusion criteria are (1) those who met the diagnostic criteria of the Chinese Guidelines for the Prevention and Treatment of Type 2 Diabetes Mellitus (2017 edition) [10] (fasting venous blood glucose $>7.0 \mathrm{mmoL} / \mathrm{L}$, or postprandial oral glucose tolerance $2 \mathrm{~h}$ venous blood glucose $>11.1 \mathrm{mmol} / \mathrm{L}$, or random venous blood glucose $>11.1 \mathrm{mmol} / \mathrm{L}$; if no symptoms of diabetes mellitus, above mentioned tests have to be repeated in the same day); (2) those who met the diagnostic criteria of PTB (met the diagnostic criteria of WS2882017 of China Health Planning Commission [11]; chest X-ray and computed tomography (CT) showed typical tuberculosis lesions; or sputum smear tested positive for acid-fast bacilli); (3) diabetes was diagnosed prior to PTB detection; (4) patients are actively on oral hypoglycemic treatment and/or insulin injections with anti-TB medication (isoniazid $(\mathrm{H})$, rifampicin $(\mathrm{R})$, pyrazinamide $(\mathrm{Z})$, and ethambutol (E) (2H R Z E/4H R [12]; a combination of $H, R, Z$, and $E$ for the first 2 months, followed by a combination of $\mathrm{H}$ and $\mathrm{R}$ for the next 4 months); (5) patients aged 18-70 years with full consciousness and voluntarily participated in the study, and who signed informed consent; (6) patients who cooperated with and insisted on the study duration and to provide complete dietary records, and who had no previous history of mental illness.

\section{Study Design}

A total of 164 patients with T2DM-PTB were randomly divided into control group and experimental group with 82 cases in each group. The two groups were on the same antiTB regimen and asked to insist on daily anti-TB medication. Sputum smear, chest X-ray, and liver and kidney functions were regularly checked. The control group adopted a self-controlled diet and the experimental group received individualized dietary interventions; both groups were on their diet for 3 months.

All enrolled patients were instructed and guided by professional trained medical stuff to complete the informed consent forms, demographic data questionnaires, NRS (nutrition risk screening) 2002 assessment forms, 3-day dietary diaries, and food frequency questionnaires, and given further explanation if the patients had any doubts. Patients in the experimental group received routine dietary nursing and health education once a week about dietary nutrition and a detail-oriented handbook.

Total daily calorie intake and proportions of three nutritional elements (sugar, protein, and fat) were calculated on the basis of individual body shape and labor strength. The conventional diabetic diet (non- individualized) was used in the control group and an individualized dietary recipe was applied in the experimental group. A detailed study algorithm is shown in Fig. 1.

\section{Intervention Program of Control Group}

The intervention program of the control group includes calculating each patient's standard weight, total daily calories, and formulating recipes. Patients can formulate specific recipes according to their own dietary preferences and they were educated about alternative dietary choices and instructed by professionally trained medical stuff.

\section{Intervention Program of Experimental Group}

\section{Investigation of Patients' Dietary Status}

Using food frequency surveys, patients in the experimental group were asked about their daily-life dietary patterns and structures; unreasonable and unhealthy dietary habits were corrected, the Dietary Health Education Manual for Type 2 Diabetes Mellitus with Tuberculosis" was delivered to each patient, and they were educated about basic knowledge of the disease. The food frequency questionnaire used in this study basically covered the types of food that Xinjiang residents usually consume and incorporated the local characteristics of Xinjiang. Uyghur foods such as steamed bread, soup rice, Naren, mixed noodles, grabbing rice, barbecue, and so on were added to the original questionnaire. 


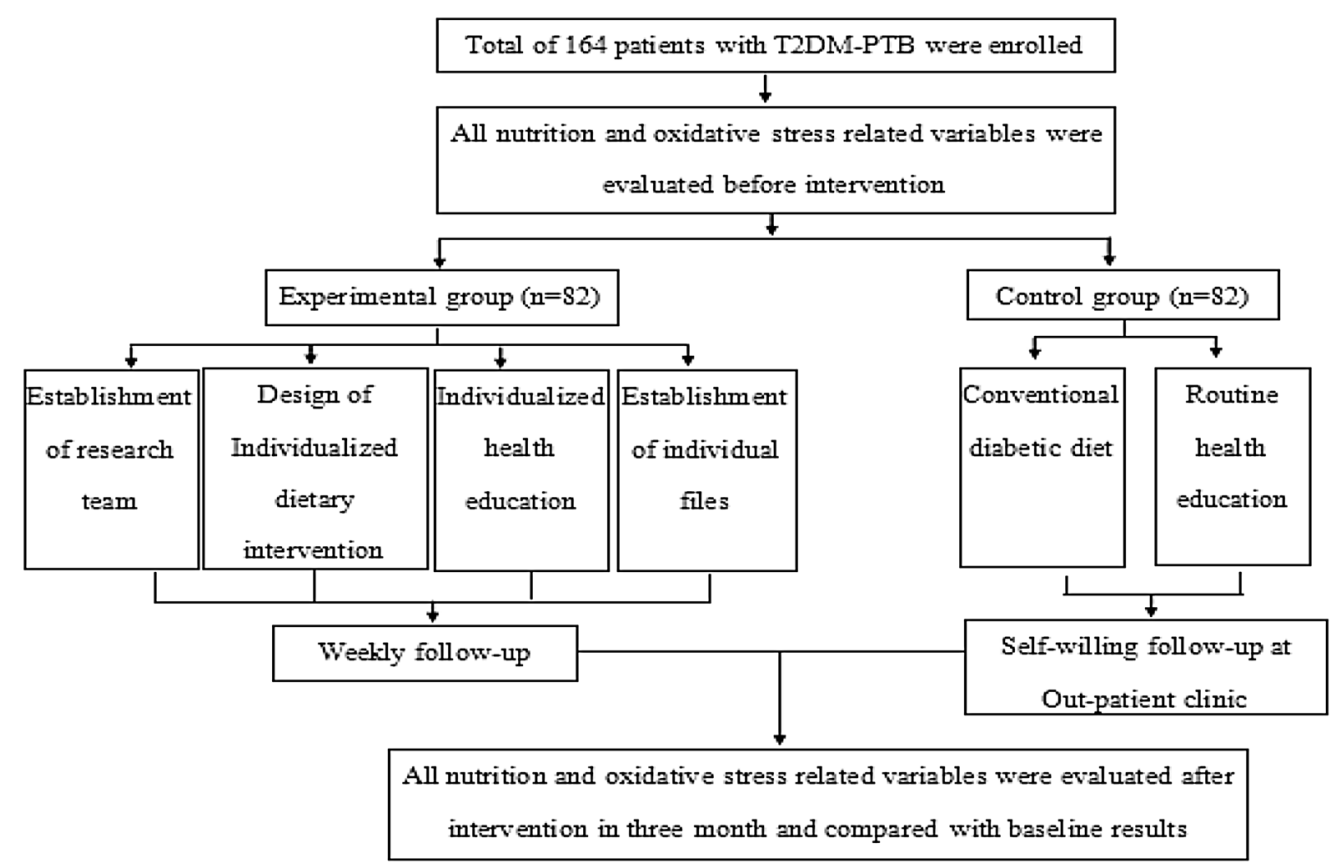

Fig. 1 Study algorithm

\section{Maintenance of Dietary Diary by Patient or Their Family Members}

Study participants were asked for dietary records of three consecutive days prior to intervention; the patients and their relatives were also trained how to record their own diets. The accuracy of these records was checked by professionally trained medical stuff. A 3-day dietary diary was used for recording (once in the week and once at the weekend). The results were averaged to reflect the daily dietary levels of the patients and to lay a foundation for individualized dietary plans for these patients.

\section{Designing Individual Quantitative Dietary Intervention Programs}

On the basis of the patient's physical measurements, nutritional and economic status, blood sugar level, individual comprehensive situation, etc., a "nutrition calculator" was used to calculate the 24-h energy intake and nutrient levels; these values were then compared with the reference dietary nutrient intake values in Chinese residents to formulate a scientific combination for each individual-a rational dietary intervention program. According to the nutritional requirements of the program, a weekly personalized quantitative diet is listed to facilitate patients' choice at home, and the diet is adjusted step by step according to the results of blood sugar monitoring.

According to TB status, the total calorie intake is slightly higher than about $10 \%$ of the diabetic calorie balance, and the amount of food exchanged daily is calculated. Carbohydrates and fats accounted for $55-65 \%$ and $20-25 \%$ of the total energy intake, respectively; an appropriate increase was observed in protein intake, accounting for more than $15 \%$ of the total energy intake $(1.5-2.0 \mathrm{~g} / \mathrm{kg}$ protein per person per day, of which high-quality protein accounted for half to two-thirds of the total protein). In order to supplement extra energy consumption due to fever and night sweats, intake of vegetables (300-500 g/day) and fruits (100-200 g/day) was suggested; to increase antioxidant and trace element intake, we suggested ingestion of fresh vegetables that are rich in various vitamins and yellow-green foods that are rich in chromium and calcium (calcium intake was higher than the recommended $800 \mathrm{mg}$ /day). While choosing food, we also referred to the glycogenesis index and load of each food item and chose some foods with 
lower glycemic index (GI) and glycemic load (GL), which is easier to grasp.

\section{Evaluation Indicators}

1. Oxidative stress indicators: superoxide dismutase (SOD), malondialdehyde (MDA), vitamin $C$, and vitamin E. Human Superoxide Dismutase (SOD) ELISA Kit, catalogue number JYM2065Hu.

Human MDA ELISA Kit, catalogue number JYM0375Hu. For the quantitative in vitro determination of active SOD in human serum, plasma, culture media, or any biological fluid. The SOD is total superoxide dismutase.

Fasting peripheral venous blood was collected in the morning and centrifuged at $1500 \times g$ for $15 \mathrm{~min}$ within $60 \mathrm{~min}$ of collection. The separated serum was stored in a refrigerator at $-80^{\circ} \mathrm{C}$. SOD, MDA, vitamin $\mathrm{C}$, and vitamin $\mathrm{E}$ detection kits were purchased from Xinjiang Yingjie Leading Biotechnology Co. Ltd.; enzyme-linked immunosorbent assays (ELISAs) were performed by strictly referring to the kit instructions. The kit involved a double-antibody one-step sandwich ELISA technique. Pre-coated antibodies that captured human SOD were coated on the micropore base; samples, standards, and horseradish peroxidase (HRP)-labeled antibodies were added in turn, after incubation and thorough washing. Tetramethylbenzidine (TMB) was used as the substrate; reaction of peroxidase with TMB produced a blue-colored compound that turned yellow on reaction with acid. The depth of color was positively correlated with human superoxide dismutase levels in the samples. Absorbance (OD) was measured at a wavelength of $450 \mathrm{~nm}$ with the enzyme-labeling instrument and sample concentrations were calculated.

2. Nutritional indicators: serum albumin, hemoglobin, total lymphocyte count (TLC), and body mass index (BMI).

3. Glycometabolism indicators: fasting blood glucose level, 2-h postprandial blood glucose level, and glycated hemoglobin level.
4. Tuberculosis control index: positive bacterial counts in the sputum.

5. C-peptide: radioimmunoassay was used to test the plasma level of C-peptide

\section{Statistical Analysis}

Statistical analysis was carried out using the SPSS 17.0 software. Continuous variables were expressed as mean \pm standard deviation. Statistical data conforming to normal distribution were tested using $t$ test of two independent samples and chi-square test. The significance level was set as $P<0.05$.

\section{RESULTS}

A total of 164 patients completed the study. Ten patients (five in each group) were lost to followup and excluded from the study. The mean (SD) age was $58.94 \pm 12.12$ years in the experiment group and $60.62 \pm 12.26$ years in the control group. There were no significant differences between the two groups with regard to age, sex, labor intensity, marital status, and course and treatment of diabetes. At baseline there were no differences between the groups in any of the study variables (Table 1 ).

All variables related to nutrition and oxidative stress were comparable between the two groups before intervention. We observed a significant elevation in ALB, Hb, TLC, BMI, SOD, VC, VE, and TCI $(P<0.05)$ and decrease in MDA, FPG, 2hPG, and HbA1c $(P<0.05)$ (Table 2).

The proportion of patients with sputum positive for bacteria was significantly lower in the experimental group than that in the control group after intervention (Table 3 ).

\section{DISCUSSION}

In this study, the incidence of hypoproteinemia and lymphopenia was higher in the two groups before intervention, suggesting that patients with T2DM-PTB have more severe visceral protein consumption. Studies have found that the 
Table 1 Comparison of demographic data between the two groups $(n=164)$

\begin{tabular}{|c|c|c|c|c|c|c|c|}
\hline \multirow[t]{2}{*}{ Index } & \multirow[t]{2}{*}{ Group } & \multicolumn{2}{|c|}{$\begin{array}{l}\text { Control group } \\
(n=82)\end{array}$} & \multicolumn{2}{|c|}{$\begin{array}{l}\text { Experimental group } \\
(n=82)\end{array}$} & \multirow[t]{2}{*}{$\chi^{2}$} & \multirow[t]{2}{*}{$P$} \\
\hline & & $n$ & $\%$ & $\bar{n}$ & $\%$ & & \\
\hline \multirow[t]{3}{*}{ Age (years) } & $18-40$ & 18 & 56.3 & 14 & 43.8 & \multirow[t]{3}{*}{1.672} & \multirow[t]{3}{*}{0.433} \\
\hline & $41-59$ & 31 & 44.3 & 39 & 55.7 & & \\
\hline & $\geq 60$ & 33 & 53.2 & 29 & 46.8 & & \\
\hline \multirow[t]{2}{*}{ Sex } & Male & 49 & 52.1 & 45 & 47.9 & \multirow[t]{2}{*}{0.399} & \multirow[t]{2}{*}{0.528} \\
\hline & Female & 33 & 47.1 & 37 & 52.9 & & \\
\hline \multirow[t]{3}{*}{ Labor intensity } & Light & 45 & 46.9 & 51 & 53.1 & \multirow[t]{3}{*}{0.995} & \multirow[t]{3}{*}{0.608} \\
\hline & Medium & 25 & 53.2 & 22 & 46.8 & & \\
\hline & Heavy & 12 & 57.1 & 9 & 42.9 & & \\
\hline \multirow[t]{2}{*}{ Marital status } & Married & 65 & 80.0 & 70 & 85.4 & \multirow[t]{2}{*}{0.837} & \multirow[t]{2}{*}{0.360} \\
\hline & Unmarried & 17 & 20.0 & 12 & 14.6 & & \\
\hline \multirow[t]{3}{*}{ Diabetes treatment } & Hypoglycemic drug & 21 & 46.7 & 24 & 53.3 & \multirow[t]{3}{*}{1.106} & \multirow[t]{3}{*}{0.575} \\
\hline & Insulin injection & 35 & 47.9 & 38 & 52.1 & & \\
\hline & Hypoglycemic drug + insulin injection & 26 & 56.5 & 20 & 43.5 & & \\
\hline \multirow[t]{3}{*}{ Disease course (years) } & $\leq 5$ & 20 & 24.4 & 23 & 28.0 & \multirow[t]{3}{*}{0.470} & \multirow[t]{3}{*}{0.791} \\
\hline & $6-10$ & 27 & 32.9 & 28 & 34.1 & & \\
\hline & $\geq 11$ & 35 & 42.7 & 31 & 37.8 & & \\
\hline
\end{tabular}

degree of nutritional deficiencies gradually increases and albumin deficiency becomes more serious as the disease progresses [13]. The overall albumin level is low because of insufficient insulin secretion in patients with diabetes, so they cannot fully utilize glucose and need to break down proteins and fats to provide calories [14]. Tuberculosis is a chronic nutrient-consumption disease; patients with TB usually experience gastrointestinal dysfunction which results in less nutrient intake and insufficient anabolism [13], but patients with TB usually also experience consumptive symptoms such as fever and night sweats which result in increased catabolism [15]. Therefore, the synthesis of albumin is reduced. Patients with T2DM-PTB experience long-term nutritional overconsumption status and loss of appetite which leads to malnutrition in the body, malnutrition causes damage to cellular immune function, which can cause a decrease in TLC and an increase in the number of $\mathrm{T}$ cells, and a decrease in the ratio of helper T cells to suppressor T cells [16]. TB lesions in the lung tissue can lead to the occurrence of infectious anemia [17]. The dietary plan formulated in this study considers not only maintaining the stability of the patient's glucose level but also ensuring adequate nutrition and correcting the possible anemia. After 3 months of individual quantitative dietary intervention and intensive health education, serum albumin, total lymphocyte count, hemoglobin, and body mass index of the experimental group were significantly higher than those in the control group, indicating that individual quantitative dietary intake can improve serum albumin level and promote lymphocyte proliferation, significantly improve 
Table 2 Comparison of variables related to nutrition and oxidative stress between the two groups before and after intervention $(\bar{x} \pm s, n=164)$

\begin{tabular}{|c|c|c|c|c|}
\hline & Control group $(n=82)$ & Experimental group $(n=82)$ & $t$ & $P$ \\
\hline \multicolumn{5}{|l|}{$\operatorname{ALB}(\mathrm{mg} / \mathrm{dL})$} \\
\hline Before intervention & $3627.00 \pm 465$ & $3533.00 \pm 478$ & 1.28 & 0.20 \\
\hline After intervention & $3829.00 \pm 371$ & $4007.00 \pm 426$ & -2.85 & 0.01 \\
\hline \multicolumn{5}{|l|}{$\mathrm{Hb}(\mathrm{mg} / \mathrm{dL})$} \\
\hline Before intervention & $11,487.00 \pm 906$ & $11,524.00 \pm 1185$ & -0.23 & 0.82 \\
\hline After intervention & $12,038.00 \pm 1134$ & $12,474.00 \pm 1272$ & -2.32 & 0.02 \\
\hline \multicolumn{5}{|l|}{$\operatorname{TLC}\left(\times 10^{9} / \mathrm{L}\right)$} \\
\hline Before intervention & $1.47 \pm 0.51$ & $1.53 \pm 0.38$ & -0.73 & 0.47 \\
\hline After intervention & $1.61 \pm 0.53$ & $1.78 \pm 0.45$ & -2.35 & 0.02 \\
\hline \multicolumn{5}{|l|}{ BMI } \\
\hline Before intervention & $22.08 \pm 0.97$ & $22.45 \pm 1.61$ & -1.79 & 0.08 \\
\hline After intervention & $22.63 \pm 0.99$ & $23.05 \pm 1.25$ & -2.40 & 0.02 \\
\hline \multicolumn{5}{|l|}{$\mathrm{SOD}(\mathrm{U} / \mathrm{ml})$} \\
\hline Before intervention & $315.03 \pm 93.42$ & $303.28 \pm 76.02$ & 0.88 & 0.38 \\
\hline After intervention & $323.40 \pm 57.34$ & $343.82 \pm 53.32$ & -2.36 & 0.02 \\
\hline \multicolumn{5}{|l|}{$\operatorname{MDA}(\mathrm{nmol} / \mathrm{ml})$} \\
\hline Before intervention & $6.45 \pm 1.77$ & $6.00 \pm 1.45$ & 1.77 & 0.08 \\
\hline After intervention & $5.85 \pm 1.70$ & $5.34 \pm 1.42$ & 2.09 & 0.04 \\
\hline \multicolumn{5}{|l|}{$\mathrm{VC}(\mu \mathrm{mol} / \mathrm{L})$} \\
\hline Before intervention & $39.24 \pm 6.09$ & $38.97 \pm 7.10$ & 0.26 & 0.80 \\
\hline After intervention & $41.45 \pm 4.85$ & $43.30 \pm 6.39$ & -2.08 & 0.04 \\
\hline \multicolumn{5}{|l|}{$\mathrm{VE}(\mu \mathrm{mol} / \mathrm{L})$} \\
\hline Before intervention & $17.23 \pm 6.51$ & $17.56 \pm 5.98$ & -0.34 & 0.74 \\
\hline After intervention & $19.19 \pm 4.74$ & $20.86 \pm 5.34$ & -2.11 & 0.04 \\
\hline \multicolumn{5}{|l|}{$\mathrm{FPG}(\mathrm{mmol} / \mathrm{L})$} \\
\hline Before intervention & $10.82 \pm 1.64$ & $10.35 \pm 1.70$ & 1.83 & 0.07 \\
\hline After intervention & $8.89 \pm 1.98$ & $8.09 \pm 1.64$ & 2.82 & 0.01 \\
\hline \multicolumn{5}{|l|}{$2 \mathrm{hPG}(\mathrm{mmol} / \mathrm{L})$} \\
\hline Before intervention & $14.53 \pm 2.72$ & $14.74 \pm 2.63$ & -0.51 & 0.61 \\
\hline After intervention & $11.43 \pm 1.94$ & $10.61 \pm 1.77$ & 2.84 & 0.01 \\
\hline \multicolumn{5}{|l|}{ HbAlc (\%) } \\
\hline Before intervention & $10.50 \pm 2.19$ & $10.38 \pm 2.05$ & 0.34 & 0.74 \\
\hline
\end{tabular}


Table 2 continued

\begin{tabular}{|c|c|c|c|c|}
\hline & Control group $(n=82)$ & Experimental group $(n=82)$ & $t$ & $\boldsymbol{P}$ \\
\hline After intervention & $8.87 \pm 1.96$ & $8.13 \pm 1.83$ & 2.51 & 0.01 \\
\hline \multicolumn{5}{|l|}{ C-peptide (ng/ml) } \\
\hline Before intervention & $1.49 \pm 0.93$ & $1.61 \pm 0.85$ & 0.86 & 0.39 \\
\hline After intervention & $1.32 \pm 0.94$ & $1.03 \pm 0.57$ & 2.39 & 0.01 \\
\hline \multicolumn{5}{|l|}{ TCI $[\mathrm{kcal} /(\mathrm{kg}$ day $)]$} \\
\hline Before intervention & $1494.71 \pm 212.18$ & $1535.02 \pm 228.27$ & 1.17 & 0.24 \\
\hline After intervention & $1691.12 \pm 245.25$ & $1784.76 \pm 252.08$ & 2.41 & 0.01 \\
\hline
\end{tabular}

$A L B$ albumin, $H b$ hemoglobin, $T L C$ total lymphocyte count, $B M I$ body mass index, $S O D$ superoxide dismutase, $M D A$ malondialdehyde, $V C$ vitamin C, $V E$ vitamin E, $F P G$ fasting plasma glucose, $2 h P G$ 2-h plasma glucose, $H b A 1 c$ glycated hemoglobin, TCI total calorie intake

Table 3 Comparison of proportion of patients with sputum positive for bacteria between the two groups before and after intervention, $n(\%)$

\begin{tabular}{llll}
\hline Group & $\boldsymbol{n}$ & Before intervention & After intervention \\
\hline Control group & 82 & $34(41.5 \%)$ & $23(28 \%)$ \\
Experimental group & 82 & $27(32.9 \%)$ & $12(14.6 \%)$ \\
$\chi^{2}$ & & 1.28 & 4.4 \\
$P$ & & 0.26 & 0.04 \\
\hline
\end{tabular}

nutritional status, and improve treatment effect. In addition, the proportion of patients with sputum positive for bacteria in the experimental group was significantly lower than that in the control group, indicating that individualized dietary intervention and intensive health education have a positive impact on disease control in patients with T2DM-PTB.

This study showed that after the intervention, the antioxidant enzyme activity in the experimental group such as SOD, VC, and VE were higher than that in the control group. Oxidative stress responses in the body are more intense as the condition of T2DM-PTB progresses and the nutritional status declines. Reasonable dietary intervention for patients with T2DM-PTB can enhance their nutritional status and reduce or avoid oxidative stress and reduce complications. As the ability of antioxidative stress is enhanced, the MDA content is significantly lower in the experimental group than that in the control group, which can remarkably improve the ability of scavenging free radicals in the body. It is fully proved that antagonizing oxidative stress is of great significance for the prevention and treatment of chronic complications of diabetes. Oxidative stress intervention is expected to become one of the new means of preventing diseases.

The level of oxidative stress in patients with pulmonary tuberculosis may increase the production of reactive oxygen species (ROS) due to the inflammatory reaction in the body [18], leading to an imbalance of oxidative stress and 
aggravation of oxidative damage. High-glucose environments can induce the production of ROS, which increases the oxidative stress of the body and aggravates the condition of DM [19]. The level of oxidative stress may be higher in patients with T2DM-PTB. The free radicals and lipids in the organism undergo peroxidation, and the final product is MDA, which can cause cross-linking polymerization of macromolecules such as proteins and nucleic acids which is cytotoxic [20]. Excessive MDA can attack DNA, protein, glycolipids, etc., causing damage and inducing various diseases [21]. This imbalance can exacerbate oxidative damage, which can lead to increased intracellular oxidative stress. It has been confirmed in human and animal experiments [22] that elevated levels of intercellular and intracellular blood glucose will lead to increased levels of oxidative stress. As pancreatic beta cells are exposed to high glucose concentrations, the islet secretion is destroyed gradually.

Studies [22, 23] have confirmed that blood glucose fluctuations in patients with diabetes are significantly associated with oxidative stress, and lowering blood glucose can enhance the ability of antioxidant systems in the body. Protection of cells can be achieved through SOD by removing excess superoxide anion radical $\left(\mathrm{O}_{2}{ }^{--}\right)$, so that they will not be destroyed under the influence of external circumstances. Fresh vegetables and fruits contain $\mathrm{VE}$ and $\mathrm{VC}$ which have antioxidant effects [24]. SOD, VE, and VC constitute an antioxidant network and these antioxidants can be regenerated and recycled through interaction, and this antioxidant network is usually activated by VE.

Compared with the control group, the fasting blood glucose, the 2-h postprandial blood glucose, and glycated hemoglobin level were significantly reduced in the experiment group after 3 months of dietary intervention which achieved our therapeutic goal. In the experimental group, dietary intervention fully considered the dual effects of drugs and food on the patient's blood glucose level. By controlling and adjusting the quality and quantity of food intake, the effect of drug interaction on blood glucose, and the intake of three nutrients were strictly calculated. Dietary intervention therapy plays an important role in controlling body mass index, improvement of islet function, regulating blood glucose and lipid levels, and can control blood glucose level by regulating genes associated with obesity. Intake of a low GI and GL diet can improve blood glucose level which rises slowly and decreases demand for insulin; long-term intake of a low GI and GL diet can reduce post-prandial intestinal hormone and insulin, slow down the rate of carbohydrate absorption [25], inhibit blood free fatty acid levels, antagonize the response of hormones, and accelerate the clearance of glucose in the blood, thereby reducing the burden of beta cells. In addition, in order to prolong the absorption time of glucose in the small intestine, it is necessary to follow a low GI and low GL diet for a long period of time, and the continuous inhibition of blood free fatty acid level and reaction to hormones can produce significant antagonism and reduce blood glucose level [26]. The results of the study confirmed that [25] a reasonable diet structure, especially after increasing the proportion of protein (average $3-5 \%$ higher than in normal people), has little effect on blood glucose level, but improves insulin sensitivity and glucose tolerance.

There was no significant difference in plasma level of C-peptide values between the two groups before intervention, and there was a significant decrease after intervention. At the same time, the plasma level of C-peptide in the experimental group was significantly lower than that of the control group. C-peptide has an anti-inflammatory effect and reduces the expression of cell adhesion molecules on the surface of vascular endothelial cells under inflammatory conditions. The combination of C-peptide and insulin can inhibit endothelial cell apoptosis induced by high glucose and improve vascular endothelium [27]. After a 3 -month personalized diet intervention program, total energy intake was increased in the experimental group, which improved the patient's dietary structure and the total energy intake, and achieved the principle of the balanced diet. 


\section{CONCLUSION}

By including a special group of patients with T2DM-PTB in Xinjiang, China, as the study population, this study examines the effects of individual dietary intervention combined with intensified health education intervention strategy; this method can integrate the language and cultural background of patients of all ethnic groups in Xinjiang as well as the psychological states of all patients and guide them in improving their dietary intake structure reasonably. Improving the level of dietary diversification and long-term quality of life of patients is of certain clinical value. In addition, patients with T2DM-PTB have better prognoses and quality of life if they receive adequate health education with proper dietary intervention and if the intervention follow-up is longer. Because of the short observation time in this study, it is impossible to further evaluate the effect of dietary intervention on the overall survival rate of the patients. Therefore, in future studies, we need to further analyze whether any other factors affect oxidant-antioxidant imbalance and malnutrition in these patients and adopt more individualized and targeted measures.

\section{ACKNOWLEDGEMENTS}

We are very grateful to our study participants for their participation.

Funding. Sponsorship for this study and the Journal's Rapid Service Fee were funded by a grant from the Natural Science Foundation of Xinjiang Uygur Autonomous Region (2015211C061).

Authorship. All named authors meet the International Committee of Medical Journal. Editors (ICMJE) criteria for authorship for this article, take responsibility for the integrity of the work as a whole, and have given their approval for this version to be published.

Disclosures. Liyun He, Guoming Zhang, Meng Wei, Yanjun Zhao, Weixing Chen,
Qiaojun Peng, and Guiyun Meng have nothing to disclose.

Compliance with Ethics Guidelines. All procedures performed in studies involving human participants were in accordance with the ethical standards of our local ethics committee (the Ethics Committee of the First Affiliated Hospital of Xinjiang Medical University) and with the 1964 Helsinki declaration and its later amendments or comparable ethical standards. Informed consent was obtained from all individual participants included in the study.

Data Availability. The datasets generated and/or analyzed during the current study are available from the corresponding author on reasonable request.

Open Access. This article is distributed under the terms of the Creative Commons Attribution-NonCommercial 4.0 International License (http://creativecommons.org/licenses/ by-nc/4.0/), which permits any noncommercial use, distribution, and reproduction in any medium, provided you give appropriate credit to the original author(s) and the source, provide a link to the Creative Commons license, and indicate if changes were made.

\section{REFERENCES}

1. Mukhtar F, Butt A. Tackling the co-epidemic of diabetes and tuberculosis: from evidence to policy and practice. J Ayub Med Coll Abbottabad. 2016;28(2):376-81.

2. Li SY, Wang SY, Li J, et al. The value of HbA1c for diagnosing type 2 diabetes mellitus between Chinese Uyghurs and Hans in Xinjiang. Int J Clin Exp Med. 2015;8(7):11352.

3. Qiang M, Kai Z, Wu Y, Chaonan C. Forecasting the incidence of tuberculosis in China using the seasonal auto-regressive integrated moving average (SARIMA) model. J Infect Public Health. 2018;11(5):707-12.

4. Chen H, He L, Cai C, et al. Characteristics of distribution of Mycobacterium tuberculosis lineages in China. Sci China. 2018;61(6):1-9. 
5. Yu J, Shen J, Wang L, et al. A case report of atypical sarcoidosis misdiagnosed as tuberculosis. Radiol Infect Dis. 2016;3(1):40-3.

6. Ravi A, Singh Sunita D, Medical GR. Global tuberculosis report 2013. Australas Med J. 2013;6(2).

7. Lönnroth K, Jaramillo E, Williams BG, et al. Drivers of tuberculosis epidemics: the role of risk factors and social determinants. Soc Sci Med. 2009;68(12):2240-6.

8. Bloomgarden ZT. Inflammation and insulin resistance. Diabetes Care. 2003;26(5):1619-23.

9. Wang Q, Ma A, Han X, et al. Hyperglycemia is associated with increased risk of patient delay in pulmonary tuberculosis in rural areas. J Diabetes. 2017;9(7):648-55.

10. Odone A, Houben RMGJ, White RG, Lönnroth K. The effect of diabetes and undernutrition trends on reaching 2035 global tuberculosis targets. Lancet Diabetes Endocrinol. 2014;2(9);754-64.

11. Lange C, Chesov D, Heyckendorf J, et al. Drug-resistant tuberculosis: an update on disease burden, diagnosis and treatment. Respirology. 2018;23(7): 656-73.

12. Guo FF. Occurrence of adverse reactions to standardized chemotherapy regimen for multidrug-resistant tuberculosis and its impact on treatment outcome. Guide China Med. 2017;15(28):137-8.

13. Yorke E, Atiase Y, Akpalu J, et al. The bidirectional relationship between tuberculosis and diabetes. Tuberc Res Treat. 2017;2017:1702578.

14. Dyson P. Erratum to: low carbohydrate diets and type 2 diabetes: what is the latest evidence. Diabetes Therapy. 2015;6(4):649.

15. Choi R, Jeong BH, Koh WJ, Lee SY. Recommendations for optimizing tuberculosis treatment: therapeutic drug monitoring, pharmacogenetics, and nutritional status considerations. Ann Lab Med. 2017;37(2):97-107.

16. Aguilera RGG, Mejia MS, Nemunaitis GA, et al. Poster 382: protein-energy malnutrition: relationship of prealbumin and bmi among individuals with spinal cord injury. PM\&R. 2010;2(9):S168.

17. Chen CY, Sheng WH, Cheng A, et al. Clinical characteristics and outcomes of Mycobacterium tuberculosis disease in adult patients with hematological malignancies. BMC Infect Dis. 2011;11(1):324.

18. Montane J, Cadavez L, Novials A. Stress and the inflammatory process: a major cause of pancreatic cell death in type 2 diabetes. Diabetes Metab Syndr Obes Targets. 2014;7:25-34.

19. Jagadish S, Hemshekhar M, Naveenkumar SK, et al. Novel oxolane derivative DMTD mitigates high glucose induced erythrocytes apoptosis by regulating oxidative stress. Toxicol Appl Pharmacol. 2017;334:167-79.

20. Savu O, Ionescu-Tirgoviste C, Atanasiu V, et al. Increase in total antioxidant capacity of plasma despite high levels of oxidative stress in uncomplicated type 2 diabetes mellitus. J Int Med Res. 2012;40(2):709-16.

21. Yunes P, Nahid K, Ebrahim S, et al. Antioxidant effects of curcuminoids in patients with type 2 diabetes mellitus: a randomized controlled trial. Inflammopharmacol. 2017;25(1):25-31.

22. Badawi A, Klip A, Haddad P, et al. Type 2 diabetes mellitus and inflammation: prospects for biomarkers of risk and nutritional intervention. Diabetes Metab Syndr Obes. 2010;3:173-86.

23. Tsai CJ, Hsieh CJ, Tung SC, et al. Acute blood glucose fluctuations can decrease blood glutathione and adiponectin levels in patients with type 2 diabetes. Diabetes Res Clin Pract. 2012;98(2):257-63.

24. Mcmacken M, Shah S. A plant-based diet for the prevention and treatment of type 2 diabetes. J Geriatr Cardiol. 2017;14(5):342-54.

25. Bajorek SA, Morello CM. Effects of dietary fiber and low glycemic index diet on glucose control in subjects with type 2 diabetes mellitus. Ann Pharmacother. 2010;44(11):1786-92.

26. Kim MJ, Kwon S, Ly SY. Effects of low glycemic index nutrition education on the blood glucose control in patients with type 2 diabetes mellitus. Korean J Nutr. 2010;43(1):46-56.

27. Leighton E, Sainsbury CA, Jones GC. A practical review of C-peptide testing in diabetes. Diab Therapy. $2017 ; 8(3): 475-87$. 